\title{
Unidirectional Flows of Binary Mixtures within the Framework of the Oberbeck-Boussinesq Model
}

\author{
V. K. Andreev ${ }^{a, b}$ and I. V. Stepanova ${ }^{a, b}$ \\ ${ }^{a}$ Institute f Computational Modelling, Siberian Branch of Russian Academy of Sciences, \\ Akademgorodok 50, str. 44, Krasnoyarsk, 660036 Russia \\ ${ }^{b}$ Siberian Federal University, Institute of Mathematics and Fundamental Informatics, \\ pr. Svobodnyi 79, Krasnoyarsk, 660041 Russia, \\ e-mail: andr@icm.krasn.ru, stepiv@icm.krasn.ru
}

Received November 11, 2015

\begin{abstract}
Existence conditions are investigated for the unidirectional flows of binary mixtures describable by the equations of motion in the Oberbeck-Boussinesq approximation with account for the thermodiffusion effect. Possible solutions are classified, known and novel classes of exact solutions being distinguished. For the solutions obtained different formulations of boundary-value problems are proposed. Flows between two rigid walls with given heat distribution laws are described.
\end{abstract}

Keywords: Oberbeck-Boussinesq model, thermodiffusion, unidirectional flow, binary mixture.

DOI: $10.1134 / \mathrm{S} 0015462816020022$

The study is devoted to investigating the thermal diffusion convection equations in the Oberbeck-Boussinesq approximation for unidirectional binary mixture flow. The latter can be realized in a channel with walls of high heat conductivity and ends different in temperature, sufficiently long in the horizontal direction. For a homogeneous fluid the mathematical model of such flow was proposed in book [1], the solution being obtained and interpreted for constant temperatures gradients at the walls in [2]. Later, this solution was obtained once more $[3,4]$ and generalized to include flows in geometrically different domains $[5,6]$. Its unsteady analogs were also considered [7, 8].

For the nonlinear dependence of density on temperature and concentration the problem of solution existence was investigated within the framework of a similar model in [9].

In the present study, the conditions of existence of the flows described are investigated in detail for a binary mixture with account for thermodiffusion. In contrast to the solutions of equations for purely thermal convection, where the linear temperature distribution at the wall requires the linearity of temperature distribution inside the layer, within the framework of the model considered the dependence of temperature and concentration on the longitudinal coordinate is generally quadratic, which in its turn suggests the quadratic law for the temperature distribution along the horizontal wall. For the system in question all possible exact solutions are exhausted by three cases, depending on the integration of the equations that link the density function and the parameters of state. For the solutions constructed, we propose some formulations of boundary-value problems for binary mixture flow between two rigid walls at which the temperature distribution is specified or there is no heat flux, as well as on the assumption that the layer is bounded from above by a free boundary. Examples are presented for two mixtures, which demonstrate the fluid behavior under the temperature gradient that acts by a given law along the horizontal walls.

It should be noted that in [10] for the system investigated the group classification problem was solved with respect to the thermal and concentration expansion coefficients, thermal diffusivity, and the diffusion and thermodiffusion parameters. The solution of that problem resulted in the list of admissible differential operators whose action leads to a reduction in the number of dependent and/or independent variables in 
the system. The knowledge of these operators enables us to construct exact solutions whose variety is presented in [11]. In [12], the group analysis was performed and an exact solution of the thermal diffusion equations with account for vibrational convection was constructed. As compared with the solutions obtained in [10-12], those obtained in the present study are, on one hand, more general and, on the other, specified for unidirectional flow alone.

\section{EQUATIONS OF THERMAL DIFFUSION MOTION AND THEIR EXACT SOLUTIONS}

The unidirectional thermal diffusion flow equations have the following form $\left(p^{*}=p^{*}(x, z), \theta=\theta(x, z)\right.$, and $c=c(x, z))$ :

$$
\begin{gathered}
\frac{1}{\rho_{0}} p_{x}^{*}=g \beta_{1} \theta+g \beta_{2} c, \quad \frac{1}{\rho_{0}} p_{z}^{*}=v w_{x x}, \\
w \theta_{z}=\chi\left(\theta_{x x}+\theta_{z z}\right), \\
w c_{z}=D\left(c_{x x}+c_{z z}\right)+D^{\theta}\left(\theta_{x x}+\theta_{z z}\right),
\end{gathered}
$$

where $\beta_{1}$ and $\beta_{2}$ are the thermal and concentration expansion coefficients, $v, \chi, D$, and $D^{\theta}$ are the viscosity, thermal diffusivity, diffusion and thermal diffusion coefficients, $p^{*}=p+g \rho_{0} x$ is the modified pressure, and $\rho_{0}$ the average density.

From the compatibility condition for Eqs. (1.1) there follow the equalities

$$
\begin{aligned}
\beta_{1} \theta+\beta_{2} c & =\alpha(x) z+\beta(x), \\
w^{\prime \prime \prime} & =\frac{g}{v} \alpha(x)
\end{aligned}
$$

with arbitrary functions $\alpha(x)$ and $\beta(x)$. Here and in what follows, by prime we denote differentiation with respect to $x$.

Eliminate from formula (1.4) the concentration $c(x, z)$

$$
c=\frac{1}{\beta_{2}}\left[-\beta_{1} \theta+\alpha(x) z+\beta(x)\right]
$$

and substitute (1.6) in Eq. (1.3). With account for expression (1.2) we arrive at the relation

$$
P w(x) \theta_{z}=\frac{D \alpha^{\prime \prime}}{\beta_{2}} z+\frac{D \beta^{\prime \prime}}{\beta_{2}}-\frac{\alpha(x)}{\beta_{2}} w
$$

where we introduced the notation

$$
P=\frac{D \beta_{1}}{\beta_{2} \chi}-\frac{\beta_{1}}{\beta_{2}}-\frac{D^{\theta}}{\chi}
$$

Assume that $P \neq 0$. Then, from (1.7) it follows that temperature is the quadratic function of the variable $z$ :

$$
\theta(x, z)=\frac{1}{P \beta_{2}}\left[\frac{D \alpha^{\prime \prime}}{2 w(x)} z^{2}+\frac{D \beta^{\prime \prime}-\alpha(x) w(x)}{w(x)} z+\gamma(x)\right]
$$

with a certain function $\gamma(x)$. The concentration field can be determined from equality (1.6) and also is the quadratic function in the variable $z$.

Calculating the derivatives $\theta_{z}, \theta_{x x}$, and $\theta_{z z}$ from formula (1.6) and substituting them in Eq. (1.2), we obtain the equality

$$
\frac{D \alpha^{\prime \prime}}{\chi} z+\frac{D \beta^{\prime \prime}-\alpha(x) w(x)}{\chi}=\frac{D \alpha^{\prime \prime}}{w(x)}+\frac{D}{2}\left(\frac{\alpha^{\prime \prime}}{w(x)}\right)^{\prime \prime} z^{2}+\left[\frac{D \beta^{\prime \prime}-\alpha(x) w(x)}{w(x)}\right]^{\prime \prime} z+\gamma^{\prime \prime},
$$

FLUID DYNAMICS Vol. $51 \quad$ No. 2016 
valid for any $z$. From this we obtain the constraint equations

$$
\begin{gathered}
\left(\frac{\alpha^{\prime \prime}}{w(x)}\right)^{\prime \prime}=0, \quad \frac{D \alpha^{\prime \prime}}{\chi}=\left[\frac{D \beta^{\prime \prime}-\alpha(x) w(x)}{w(x)}\right]^{\prime \prime}, \\
\gamma^{\prime \prime}=\frac{D \beta^{\prime \prime}-\alpha(x) w(x)}{\chi}-\frac{D \alpha^{\prime \prime}}{w(x)} .
\end{gathered}
$$

The first and second equations from system (1.9) can be integrated in the form

$$
\begin{gathered}
\alpha^{\prime \prime}=\left(a_{0} x+a_{1}\right) w(x), \\
D \beta^{\prime \prime}=\left[\left(1+\frac{D}{\chi}\right) \alpha(x)-b_{0} x-b_{1}\right] w(x)
\end{gathered}
$$

with constant $a_{0}, a_{1}, b_{0}$, and $b_{1}$. The third equation from (1.9) then takes the form

$$
\gamma^{\prime \prime}=\left[\frac{D}{\chi} \alpha(x)-b_{0} x-b_{1}\right] \frac{w(x)}{\chi}-D\left(a_{0} x+a_{1}\right) .
$$

Differentiating Eq. (1.5) twice with respect to $x$ and using formula (1.10), we obtain the equation for $w(x)$

$$
w^{(\mathrm{V})}-\frac{g}{v}\left(a_{0} x+a_{1}\right) w=0 .
$$

Thus, from the determined velocity $w(x)$ and expression (1.10) we find $\alpha(x)$ and then, from relations (1.11) and (1.12), find in quadratures the functions $\beta(x)$ and $\gamma(x)$ and, hence, temperature and concentration. Pressure can be determined from Eq. (1.1) and the problem is completely solvable.

Note that the solution of Eqs. (1.10)-(1.13) depends on the constants $a_{0}$ and $a_{1}$. Consider in detail three (I-III) cases.

I. If $a_{0}=a_{1}=0$, then from (1.10) $\alpha=\alpha_{0} x+\alpha_{1}$ and from the second and third equations in (1.9)

$$
\beta^{\prime \prime}=\left(b_{0} x+b_{1}\right) w(x), \quad \gamma^{\prime \prime}=\left(\gamma_{0} x+\gamma_{1}\right) w(x),
$$

where $\gamma_{0}=\left(D b_{0}-\alpha_{0}\right) / \chi$ and $\gamma_{1}=\left(D b_{1}-\alpha_{1}\right) / \chi$. The velocity function can be determined from equality (1.5)

$$
w=\frac{g}{v}\left(\frac{\alpha_{0} x^{4}}{24}+\frac{\alpha_{1} x^{3}}{6}+\frac{C_{1} x^{2}}{2}+C_{2} x+C_{3}\right) .
$$

The functions $\beta$ and $\gamma$ then are seventh-power polynomials:

$$
\begin{gathered}
\beta(x)=\frac{g}{v}\left[\frac{\alpha_{0} b_{0} x^{7}}{1008}+\frac{1}{720}\left(4 b_{0} \alpha_{1}+\alpha_{0} b_{1}\right) x^{6}+\frac{1}{120}\left(b_{1} \alpha_{1}+3 C_{1} b_{0}\right) x^{5}\right. \\
\left.+\frac{1}{24}\left(2 b_{0} C_{3}+b_{1} C_{1}\right) x^{4}+\frac{1}{6}\left(\alpha_{0} C_{3}+b_{1} C_{2}\right) x^{3}+\frac{b_{1} C_{3}}{2} x^{2}+C_{4} x+C_{5}\right], \\
\gamma(x)=\frac{g}{v}\left[\frac{\alpha_{0} \gamma_{0} x^{7}}{1008}+\frac{1}{720}\left(4 \gamma_{0} \alpha_{1}+\alpha_{0} \gamma_{1}\right) x^{6}+\frac{1}{120}\left(\gamma_{1} \alpha_{1}+3 C_{1} \gamma_{0}\right) x^{5}\right. \\
\left.+\frac{1}{24}\left(2 \gamma_{0} C_{3}+b_{1} C_{1}\right) x^{4}+\frac{1}{6}\left(\alpha_{0} C_{3}+\gamma_{1} C_{2}\right) x^{3}+\frac{\gamma_{1} C_{3}}{2} x^{2}+C_{6} x+C_{7}\right] .
\end{gathered}
$$

In formulas (1.14)-(1.16), $\alpha_{0}, \alpha_{1}$, and $C_{i}(i=1, \ldots, 7)$ are arbitrary constants.

In this case, temperature and concentration are functions linear in $z$ and can be determined using (1.15) and (1.16) by the formulas

$$
\theta=\frac{1}{P \beta_{2}}\left(\gamma(x)+\chi\left(\gamma_{0} x+\gamma_{1}\right) z\right)
$$




$$
c=\frac{1}{\beta_{2}}\left[\beta(x)-\frac{\beta_{1}}{P \beta_{2}} \gamma(x)+\chi\left(\left(\alpha_{0}-\frac{\chi \beta_{1}}{P \beta_{2}} \gamma_{0}\right) x+\alpha_{1}-\frac{\beta_{1} \chi}{P \beta_{2}} \gamma_{1}\right) z\right] .
$$

This solution was first considered in [4]. In [13], solution (1.14), (1.17), (1.18) was used for describing the regime of two-layer flow with evaporation at the interface between liquid and gas phases.

II. If $a_{0}=0$ and $a_{1}>0$, then the general solution of Eq. (1.13) has the form

$$
\begin{gathered}
w(x)=c_{1} \exp (\lambda x)+\exp \left(\lambda \mu_{1} x\right)\left(c_{2} \cos \lambda \mu_{2} x+c_{3} \sin \lambda \mu_{2} x\right) \\
+\exp \left(\lambda \mu_{3} x\right)\left(c_{4} \cos \lambda \mu_{4} x+c_{5} \sin \lambda \mu_{4} x\right), \\
\lambda=\left(\frac{g a_{1}}{v}\right)^{1 / 5}, \quad \mu_{1}=\frac{1}{4}(\sqrt{5}-1), \quad \mu_{2}=\frac{1}{4} \sqrt{10+2 \sqrt{5}}, \\
\mu_{3}=-\frac{1}{4}(\sqrt{5}+1), \quad \mu_{4}=\frac{1}{4} \sqrt{10-2 \sqrt{5}},
\end{gathered}
$$

where $c_{j}(j=1, \ldots, 5)$ are constants.

Note that the roots of the characteristic equation $\mu_{i}(i=1, \ldots, 4)$ have the following interesting properties to be used in what follows for calculations:

$$
\mu_{1}^{2}+\mu_{2}^{2}=1, \quad \mu_{3}^{2}+\mu_{4}^{2}=1, \quad \mu_{1}^{2}-\mu_{2}^{2}=\mu_{3}, \quad \mu_{3}^{2}-\mu_{4}^{2}=\mu_{1}
$$

Reproduce expressions for the functions $\alpha, \beta$, and $\gamma$ :

$$
\begin{gathered}
\alpha(x)=p_{1} \exp (\lambda x)+\exp \left(\lambda \mu_{1} x\right)\left(p_{2} \cos \lambda \mu_{2} x+p_{3} \sin \lambda \mu_{2} x\right) \\
+\exp \left(\lambda \mu_{3} x\right)\left(p_{4} \cos \lambda \mu_{4} x+p_{5} \sin \lambda \mu_{4} x\right)+c_{6} x+c_{7}, \\
\beta(x)=B^{1}(x), \quad \gamma(x)=B^{2}(x), \\
B^{i}(x)=q_{1}^{i} \exp (2 \lambda x)+\exp \left(\lambda\left(1+\mu_{1}\right) x\right)\left(q_{2}^{i} \cos \lambda \mu_{2} x+q_{3}^{i} \sin \lambda \mu_{2} x\right)+\exp \left(\lambda\left(1+\mu_{3}\right) x\right) \\
\times\left(q_{4}^{i} \cos \lambda \mu_{4} x+q_{5}^{i} \sin \lambda \mu_{4} x\right)+\exp \left(\lambda\left(\mu_{1}+\mu_{3}\right) x\right)\left(q_{6}^{i} \cos \lambda\left(\mu_{2}-\mu_{4}\right) x\right. \\
\left.+q_{7}^{i} \sin \lambda\left(\mu_{2}-\mu_{4}\right) x+q_{8}^{i} \cos \lambda\left(\mu_{2}+\mu_{4}\right) x+q_{9}^{i} \sin \lambda\left(\mu_{2}+\mu_{4}\right) x\right) \\
+\exp \left(2 \lambda \mu_{1} x\right)\left(q_{10}^{i}+q_{11}^{i} \cos 2 \lambda \mu_{2} x+q_{12}^{i} \sin 2 \lambda \mu_{2} x\right) \\
+\exp \left(2 \lambda \mu_{3} x\right)\left(q_{13}^{i}+q_{14}^{i} \cos 2 \lambda \mu_{4} x+q_{15}^{i} \sin 2 \lambda \mu_{4} x\right) \\
+\exp (\lambda x)\left(q_{16}^{i} x+q_{17}^{i}\right)+\exp \left(\lambda \mu_{1} x\right)\left(\left(q_{18}^{i} x+q_{19}^{i}\right) \cos \lambda \mu_{2} x\right. \\
\left.+\left(q_{20}^{i} x+q_{21}^{i}\right) \sin \lambda \mu_{2} x\right)+\exp \left(\lambda \mu_{3} x\right)\left(\left(q_{22}^{i} x+q_{23}^{i}\right) \cos \lambda \mu_{4} x\right. \\
\left.+\left(q_{24}^{i} x+q_{25}^{i}\right) \sin \lambda \mu_{4} x\right)+c_{q^{i}}^{i} x+c_{s}^{i} .
\end{gathered}
$$

In formulas (1.20)-(1.22) the values $q_{1}^{i}, \ldots, q_{25}^{i}(i=1,2)$ are nonlinear combinations of the constants $c_{1}, \ldots, c_{7}$, the coefficients of the characteristic equation $\mu_{1}, \ldots, \mu_{4}$, and the physical constants $\lambda$, $\chi$, and $D$. The constants $c_{q}^{i}$ and $c_{s}^{i}$ have the form $c_{q}^{1}=c_{8}, c_{s}^{1}=c_{9}, c_{q}^{2}=c_{10}, c_{s}^{2}=c_{11}$. The constants $p_{i}(i=1, \ldots, 5)$ can be determined by the formulas

$$
\begin{gathered}
p_{1}=\frac{a_{1} c_{1}}{\lambda^{2}}, \quad p_{2}=\frac{a_{1}\left(c_{2} \mu_{3}-2 \mu_{1} \mu_{2} c_{3}\right)}{\lambda^{2}}, \quad p_{3}=\frac{a_{1}\left(2 c_{2} \mu_{1} \mu_{2}+\mu_{3} c_{3}\right)}{\lambda^{2}}, \\
p_{4}=\frac{a_{1}\left(c_{4} \mu_{1}-2 \mu_{3} \mu_{4} c_{5}\right)}{\lambda^{2}}, \quad p_{5}=\frac{a_{1}\left(2 c_{4} \mu_{3} \mu_{4}+\mu_{1} c_{5}\right)}{\lambda^{2}} .
\end{gathered}
$$

III. For $a_{0}>0$, Eq. (1.13) can be reduced by the change of the variable $y=\left(g\left(a_{0} x+a_{1}\right)\right) / v$ to the following equation: 


$$
w^{(\mathrm{V})}=A y w,
$$

where $A=\left(v /\left(g a_{0}\right)\right)^{5}$ and the derivative is calculated with respect to the variable $y$.

In accordance with [14], the solution of the transformed equation (1.23) has the form

$$
\begin{gathered}
w(y)=\sum_{k=0}^{5} s_{k} \varepsilon_{k} \int_{0}^{\infty} \exp \left(\varepsilon_{k} y t-\frac{t^{6}}{6 A}\right) d t, \\
\varepsilon_{k}=\exp \frac{\pi k i}{3}, \quad \sum_{k=0}^{5} s_{k}=0,
\end{gathered}
$$

where $i$ is the imaginary unit.

For example, the second term in the sum (1.24) can be written as

$$
\frac{s_{1}(1+i \sqrt{3})}{4} \int_{0}^{\infty} \exp \left((1+i \sqrt{3}) y t-\frac{t^{6}}{3 A}\right) d t
$$

and is in calculating the integral the hypergeometric function. The temperature and concentration functions can be reconstructed after expressions for $\alpha, \beta$, and $\gamma$ are found. The expressions are cumbersome and, therefore, in this case the solution is not reproduced explicitly.

If the constant $P$ from formula (1.7) is equal to zero, the following equality is valid:

$$
D^{\theta}=\frac{\beta_{1}}{\beta_{2}}(D-\chi) \text {. }
$$

We do not know such binary mixtures whose physical properties satisfy this relationship exactly. Therefore, in what follows this case will only be discussed within the framework of the mathematical solution of the problem formulated. If the equality $P=0$ is valid, this leads to the linear dependence of the function $\alpha$ on $x$. From Eq. (1.5) the velocity function can be found in the form of a fourth-power polynomial and the function $\beta$ is a seventh-power polynomial. The temperature field can be determined as the solution of the elliptic equation (1.2) and the concentration by formula (1.6).

\section{FORMULATION OF BOUNDARY-VALUE PROBLEMS FOR $P \neq 0$}

Let the binary mixture flow unidirectionally within a plane layer of thickness $L$. Assign boundary conditions for Eq. (1.13). At the rigid walls the no-slip conditions are satisfied:

$$
w(0)=w(L)=0 .
$$

Moreover, the mixture flow rate across the layer cross-section at the point $z=0$ is given, that is,

$$
\int_{0}^{L}(1-\beta(x)) w(x) d x=q=\text { const. }
$$

The latter equality follows from the fact that $\rho=\rho_{0}\left(1-\beta_{1} \theta-\beta_{2} c\right)=\rho_{0}(1-\beta(x))$ by virtue of relation (1.4) ( $q$ is the known constant). Note that instead of (2.2) we can assign the condition

$$
\int_{0}^{L} w(x) d x=0
$$


Equality (2.3) follows from the mass conservation equation at constant average mixture density. Further conditions depend on the formulation of the problem for temperature and concentration.

2.1. Flow in the layer with the given temperature at the walls. The form of the function $\theta$ from equality (1.8) predetermines the wall temperature distribution quadratic in $z$

$$
\begin{gathered}
\left.\theta\right|_{x=0}=f_{0} z^{2}+f_{1} z+f_{2}, \\
\left.\theta\right|_{x=L}=h_{0} z^{2}+h_{1} z+h_{2},
\end{gathered}
$$

where $f_{j}$ and $h_{j}(j=0,1,2)$ are the given constants.

The conditions formulated enable us to determine the constants $a_{0}, a_{1}, b_{0}$, and $b_{1}$ and to obtain conditions for the function $\gamma$ :

$$
\begin{gathered}
a_{0}=\frac{2 P \beta_{2}}{D L}\left(h_{0}-f_{0}\right), \quad a_{1}=\frac{2 P \beta_{2}}{D} f_{0}, \\
b_{0}=\frac{1}{L}\left[\frac{D}{\chi}(\alpha(L)-\alpha(0))+P \beta_{2}\left(f_{1}-h_{1}\right)\right], \quad b_{1}=\frac{D \alpha(0)}{\chi}-P \beta_{2} f_{1}, \\
\gamma(0)=P \beta_{2} f_{2}, \quad \gamma(L)=P \beta_{2} h_{2} .
\end{gathered}
$$

Since the walls are assumed to be insoluble, there are no mass fluxes across them. This can be expressed by the conditions

$$
\left.\left(D c_{x}+D^{\theta} \theta_{x}\right)\right|_{x=0}=0,\left.\quad\left(D c_{x}+D^{\theta} \theta_{x}\right)\right|_{x=L}=0 .
$$

In accordance with (1.6), (1.8), (1.10), and (1.11), we have

$$
\begin{gathered}
D c_{x}+D^{\theta} \theta_{x}=\left(D^{\theta}-\frac{\beta_{1}}{\beta_{2}} D\right) \theta_{x}+\frac{D}{\beta_{2}} \alpha^{\prime} z+\frac{D}{\beta_{2}} \beta^{\prime} \\
=\left(D^{\theta}-\frac{\beta_{1}}{\beta_{2}} D\right) \frac{1}{P \beta_{2}}\left[\frac{D}{2} a_{0} z^{2}+\left(\frac{D}{\chi} \alpha^{\prime}-b_{0}\right) z+\gamma^{\prime}\right]+\frac{D}{\beta_{2}} \alpha^{\prime} z+\frac{D}{\beta_{2}} \beta .
\end{gathered}
$$

Therefore, if $P_{1}=D^{\theta}-\beta_{1} D / \beta_{2} \neq 0$, then in (2.6) $a_{0}=0\left(h_{0} \equiv f_{0}\right)$,

$$
\begin{gathered}
\beta^{\prime}(0)=-\frac{P_{1}}{P D} \gamma^{\prime}(0), \quad \beta^{\prime}(L)=-\frac{P_{1}}{P D} \gamma^{\prime}(L), \\
\alpha^{\prime}(0)=\alpha^{\prime}(L)=-\frac{\beta_{2} P_{1}}{\beta_{1} D} b_{0} .
\end{gathered}
$$

Using Eq. (1.5) for $a_{0}=0$ and the third formula (2.6), we find two lacking conditions for the function $w(x)$

$$
\begin{gathered}
w^{(\mathrm{IV})}(0)=w^{(\mathrm{IV})}(L), \\
w^{(\mathrm{IV})}(0)+\frac{\beta_{2} P_{1}}{\chi \beta_{1} L}\left[w^{\prime \prime \prime}(L)-w^{\prime \prime \prime}(0)\right]=\frac{g \beta_{2}^{2} P_{1}}{v D \beta_{1} L} P\left(h_{1}-f_{1}\right) .
\end{gathered}
$$

For $P_{1}=0$, the following relation holds:

$$
D^{\theta}=\frac{\beta_{1}}{\beta_{2}} D
$$

This means that from equality (2.8) the boundary conditions can be rewritten as

$$
\alpha^{\prime}(0)=0, \quad \alpha^{\prime}(L)=0, \quad \beta^{\prime}(0)=0, \quad \beta^{\prime}(L)=0 .
$$


All conditions (2.6) are assumed to be satisfied. In this case, equalities (2.10) and (2.11) for the function $w(x)$ can be reduced to the form

$$
w^{(\mathrm{IV})}(0)=0, \quad w^{(\mathrm{IV})}(L)=0 .
$$

2.2. Flow in the layer with the given temperature of the lower wall and the thermally insulated upper wall. Now, at the lower wall conditions (2.4) and the first condition from (2.7) are satisfied. From these conditions there follows that

$$
\begin{gathered}
a_{1}=\frac{2 P \beta_{2}}{D} f_{0}, \quad b_{1}=\frac{D \alpha(0)}{\chi}-P \beta_{2} f_{1}, \quad \gamma(0)=P \beta_{2} f_{2}, \\
a_{0}=0, \quad \beta^{\prime}(0)=-\frac{P_{1}}{D} \gamma^{\prime}(0), \quad \alpha^{\prime}(0)=-\frac{\beta_{2}}{\beta_{1}} \frac{P_{1}}{D} b_{0} .
\end{gathered}
$$

The thermal insulation condition for the upper wall means that $\left.\theta_{x}\right|_{x=L}=0$. Then, from the second condition in (2.7), there follows that $\left.c_{x}\right|_{x=L}=0$ too. In its turn, this yields the equalities

$$
\alpha^{\prime}(L)=0, \quad \beta^{\prime}(L)=0, \quad \gamma^{\prime}(L)=0, \quad b_{0}=0 .
$$

From (2.13) and (2.14) we can derive $\left(P_{1} \neq 0\right)$

$$
\begin{gathered}
a_{0}=0, \quad a_{1}=\frac{2 P \beta_{2}}{D} f_{0}, \quad b_{0}=0, \quad b_{1}=\frac{D \alpha(0)}{\chi}-P \beta_{2} f_{1}, \\
\gamma(0)=P \beta_{2} f_{2}, \quad \beta^{\prime}(0)=\frac{P_{1}}{D} \gamma^{\prime}(0), \quad \beta^{\prime}(L)=0, \\
\alpha^{\prime}(0)=0, \quad \alpha^{\prime}(L)=0 .
\end{gathered}
$$

In this case, for $w(x)$, in addition to (2.1) and (2.2), conditions (2.12) are satisfied.

If $P_{1}=0$, then

$$
\alpha^{\prime}(0)=0, \quad \beta^{\prime}(0)=0,
$$

The constants $a_{0}, a_{1}, b_{1}$, and $\gamma(0)$ are the same as in (2.6). Moreover, equalities (2.13) are satisfied. Thus, for $w(x)$ boundary conditions (2.12) are valid.

2.3. Flow in the layer with two thermally insulated walls. Since in this case $\theta_{x}=0$ and $c_{x}=0$ at $x=0$ and $x=L$, from (1.6) and (1.8) it follows that

$$
\alpha^{\prime}(0)=\alpha^{\prime}(L)=0, \quad \beta^{\prime}(0)=\beta^{\prime}(L)=0, \quad a_{0}=0, \quad b_{0}=0, \quad \gamma^{\prime}(0)=\gamma^{\prime}(L)=0
$$

For $w(x)$ conditions (2.12) are satisfied. Note that here there are no restrictions on the two constants $a_{1}$ and $b_{1}$ which cannot be determined within the framework of the problem formulation adopted. We can propose the following solution method. Replacing the function $\alpha(x)$ by $\alpha^{*}(x)=\alpha(x)-\chi b_{1} / D$, we can set $b_{1}=0$. If assuming in (2.2) $q=0$, for the velocity $w(x)$ we obtain the spectral problem. The parameter $a_{1}$ can be determined from the solution non-triviality condition for the spectral problem obtained. It should be noted that in the general case we cannot set $b_{1}=0$ since the problem then becomes overdetermined.

2.4. Flow in the layer with the undeformable free upper boundary. Here, instead of (2.1), we have

$$
w(0)=0, \quad w^{\prime}(L)=0,
$$

and the true pressure

$$
p=g \rho_{0}\left[L-x+\int_{L}^{x} \beta(x) d x+z \int_{L}^{x} \alpha(x) d x\right]+p_{a}
$$


so that $p=p_{a}$ at $x=L$, where $p_{a}=$ const is the atmospheric pressure. For temperature, at the free boundary $x=L$ the following condition is satisfied:

$$
k \theta_{x}+\sigma\left(\theta-\theta_{\text {out }}\right)=Q,
$$

where $k$ is the thermal conductivity of the mixture, $\sigma$ the heat-transfer coefficient, $\theta_{\text {out }}$ the surrounding medium temperature, and $Q$ the given heat flux. Boundary conditions (2.7) remain unchanged. Assume that at the lower rigid wall the condition for temperature (2.4) is satisfied. Therefore, $a_{1}, b_{1}$, and $\gamma(0)$ can be expressed by formulas (2.13). If $P_{1} \neq 0$, then $a_{0}=0$ and conditions (2.9) are valid. Relation (2.17) yields the equalities

$$
\begin{gathered}
a_{0}(k+\sigma L)+\sigma a_{1}=\frac{2 P \beta_{2}}{D}\left(Q_{0}+\sigma h_{\text {out }}^{0}\right), \\
k\left(\frac{D}{\chi} \alpha^{\prime}(L)-b_{0}\right)+\sigma\left(\frac{D}{\chi} \alpha(L)-b_{0} L-b_{1}\right)=P \beta_{2}\left(Q_{1}+\sigma h_{\text {out }}^{1}\right), \\
k \gamma^{\prime}(L)+\sigma \gamma(L)=P \beta_{2}\left(Q_{2}+\sigma h_{\text {out }}^{2}\right) .
\end{gathered}
$$

It is thus assumed that

$$
\theta_{\text {out }}=h_{\text {out }}^{0} z^{2}+h_{\text {out }}^{1} z+h_{\text {out }}^{2}, \quad Q=Q_{0} z^{2}+Q_{1} z+Q_{2},
$$

where $h_{\text {out }}^{j}$ and $Q_{j}(j=0,1,2)$ are constants.

Hence,

$$
\begin{gathered}
a_{0}=\frac{2 P \beta_{2}}{D(k+\sigma L)}\left(Q_{0}+\sigma h_{\text {out }}^{0}-\sigma f_{0}\right) \\
b_{0}=\frac{1}{k+\sigma L}\left[P \beta_{2}\left(\sigma f_{1}-Q_{1}-\sigma h_{\text {out }}^{1}\right)+\frac{D}{\chi}\left(k \alpha^{\prime}(L)+\sigma \alpha(L)-\sigma \alpha(0)\right)\right] .
\end{gathered}
$$

Since $a_{0}=0$,

$$
Q_{0}=\sigma\left(f_{0}-h_{\text {out }}^{0}\right) \text {. }
$$

The last equality in (2.9) and (2.19) yield the relation

$$
k \alpha^{\prime}(L)+\sigma \alpha(L)-\sigma \alpha(0)+\frac{\beta_{1}}{\beta_{2}} \frac{\chi}{P_{1}}(k+\sigma L) \alpha^{\prime}(0)=\frac{P \beta_{2} \chi}{D}\left[Q_{1}+\sigma\left(h_{\mathrm{out}}^{1}-f_{1}\right)\right] .
$$

Thus, the boundary conditions for $w(x)$ consist of equalities (2.2), (2.11), and (2.16) and the following equality that follows from (1.5) and (2.20):

$$
k w^{(\mathrm{IV})}(L)+\sigma w^{\prime \prime \prime}(L)-\sigma w^{\prime \prime \prime}(0)+\frac{\beta_{1}}{\beta_{2}} \frac{\chi}{P_{1}}(k+\sigma L) w^{(\mathrm{IV})}(0)=\frac{P \beta_{2} \chi g}{D v}\left[Q_{1}+\sigma\left(h_{\mathrm{out}}^{1}-f_{1}\right)\right] .
$$

\section{EXAMPLES OF SOLUTIONS OF BOUNDARY VALUE PROBLEMS}

Linear temperature distribution over the walls. Consider the solution of Eqs. (1.1)-(1.3) for the linear form of the function $\alpha$ of $x$ from (1.4), which is determined by formulas (1.14), (1.17), and (1.18). Note that in this case the temperature and concentration functions are linear in $z$. This means that, when solving the problem of binary mixture flow between two rigid walls with boundary conditions (2.4), (2.5), we must set $f_{0}$ and $h_{0}$ to be equal to zero and, thus, $a_{0}=0$. In addition to the conditions for velocity (2.1) and (2.2) and for temperature and concentration (2.4), (2.5), and (2.7), we will specify the average concentration in the layer cross-section $z=0$

$$
\frac{1}{L} \int_{0}^{L} c(x) d x=c_{0}=\text { const. }
$$

FLUID DYNAMICS Vol. $51 \quad$ No. 2016 


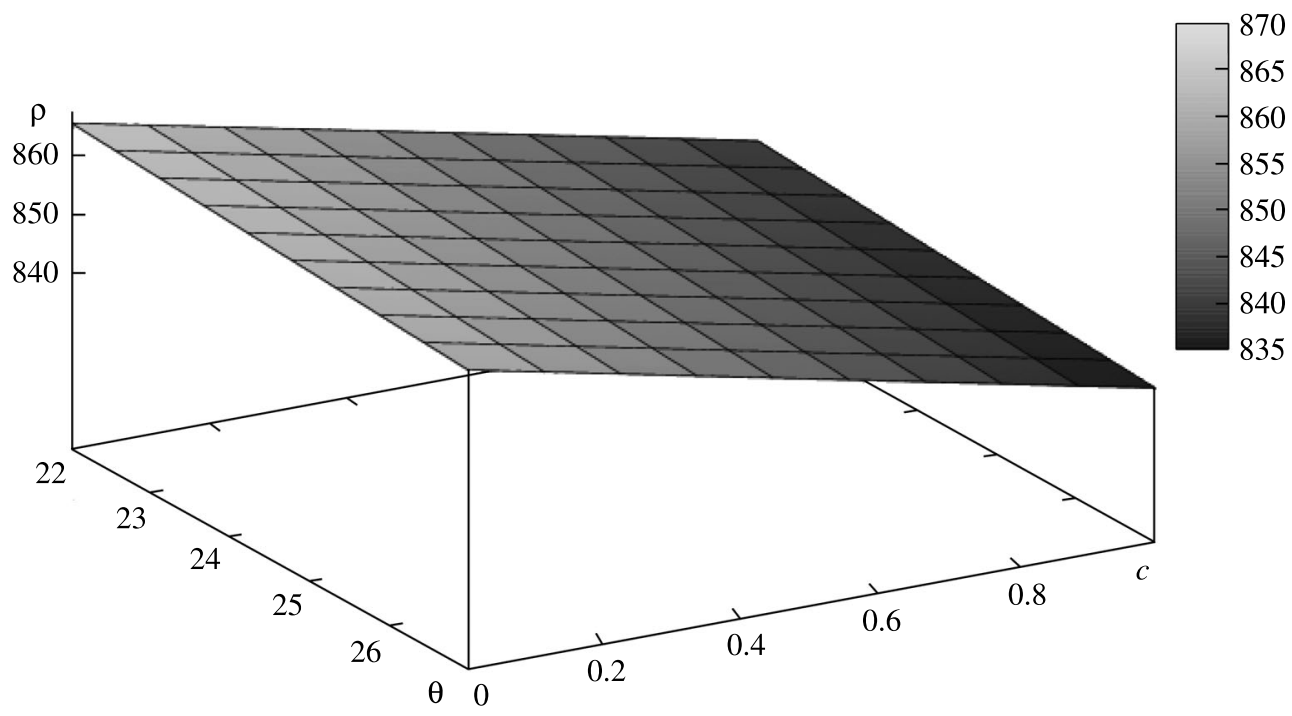

Fig. 1. Density profile for $70 \%$ isopropanol aqueous solution.

Note that the conditions listed yield eleven equations for the constants $\alpha_{0}, \alpha_{1}, b_{0}, b_{1}, C_{1}, \ldots, C_{7}$. The problem is formulated correctly, can be solved completely, and for $70 \%$ propanol aqueous solution its solution is shown in Figs. 2-4. The physical characteristics of the mixture at average temperature $25^{\circ} \mathrm{C}$ and average isopropanol concentration $c_{0}=0.7$ were calculated from the data given in [15] and are presented in Table 1.

In Figs. 1 and 2 the coordinate dependence of the mixture density and velocity is presented. Since the thermal diffusion coefficient is positive, anomalous thermodiffusion takes place: the light component (isopropanol) is concentrated near the less heated wall $x=0$, which is demonstrated by the velocity graph.

In Figs. 3 and 4 the dependence of the mixture temperature and concentration on the coordinates are presented on the assumption that $x$ and $z$ vary on the range from 0 to 1 . It should be noted that the flow regime obtained is only possible for a relatively small temperature gradient of about $5^{\circ} \mathrm{C}$ for the initial temperature equal to $25^{\circ} \mathrm{C}$. The light component concentration then varies from 0.5 to 0.9 at the initial average concentration 0.7 .

Parabolic temperature distribution over the walls. Consider the solution of Eqs. (1.1)-(1.3) with velocity given by formula (1.19) and temperature and concentration determined using relations (1.20)-(1.22). We will use this solution for describing the flow between two rigid walls the upper of which is thermally insulated (see Sect. 2, (2.13)-(2.15)). In boundary condition (2.4) we will set $f_{1}=0$.

Introduce the dimensionless parameters

$$
\begin{gathered}
w^{*}=\frac{w L}{v}, \quad \theta^{*}=\frac{\theta}{\theta_{0}}, \quad x^{*}=\frac{x}{L}, \quad z^{*}=\frac{z}{L}, \quad \alpha^{*}=\alpha L, \\
a_{1}^{*}=a_{1} v L^{2}, \quad f_{0}^{*}=\frac{f_{0} L^{2}}{\theta_{0}}, \quad f_{2}^{*}=\frac{f_{2}}{\theta_{0}},
\end{gathered}
$$

where $L$ is the characteristic linear dimension and $\theta_{0}$ the average mixture temperature.

In what follows all calculations will be performed for the dimensionless quantities in whose notation the asterisk is omitted. Using formulas (1.19) and (1.20), boundary conditions (2.1), (2.3), and (2.12), and

Table 1

\begin{tabular}{c|c|c|c|c|c|c}
\hline$\rho_{0}, \mathrm{~kg} / \mathrm{m}^{3}$ & $v, \mathrm{~m}^{2} / \mathrm{s}$ & $\beta_{1}, 1 / \mathrm{K}$ & $\beta_{2}$ & $\chi, \mathrm{m}^{2} / \mathrm{s}$ & $D, \mathrm{~m}^{2} / \mathrm{s}$ & $D^{\theta}, \mathrm{m}^{2} /(\mathrm{Ks})$ \\
\hline 885.03 & $2745 \times 10^{-8}$ & $1005 \times 10^{-6}$ & $2789 \times 10^{-5}$ & $689 \times 10^{-10}$ & $245 \times 10^{-12}$ & $1010 \times 10^{-15}$ \\
\hline
\end{tabular}




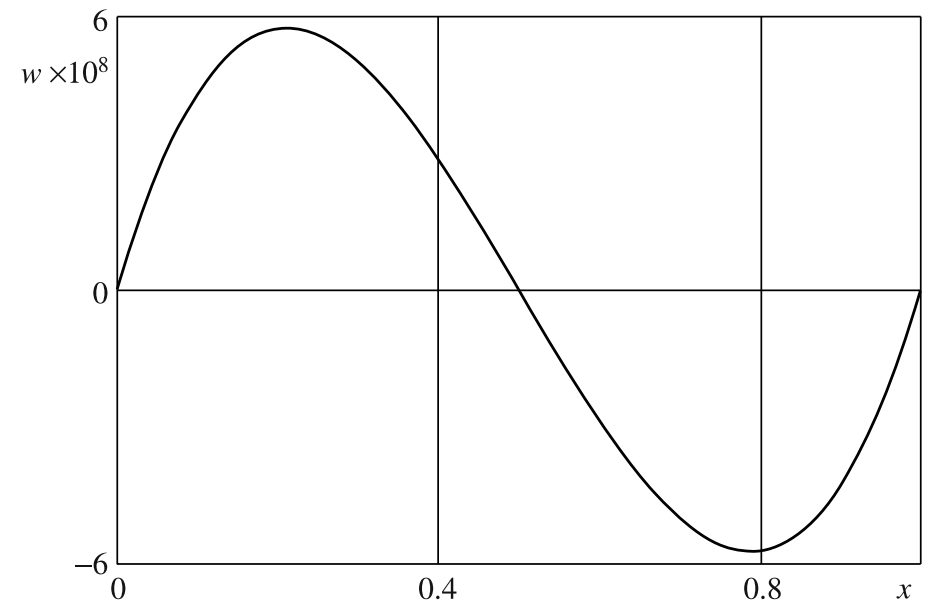

Fig. 2. Velocity profile for $70 \%$ isopropanol aqueous solution.

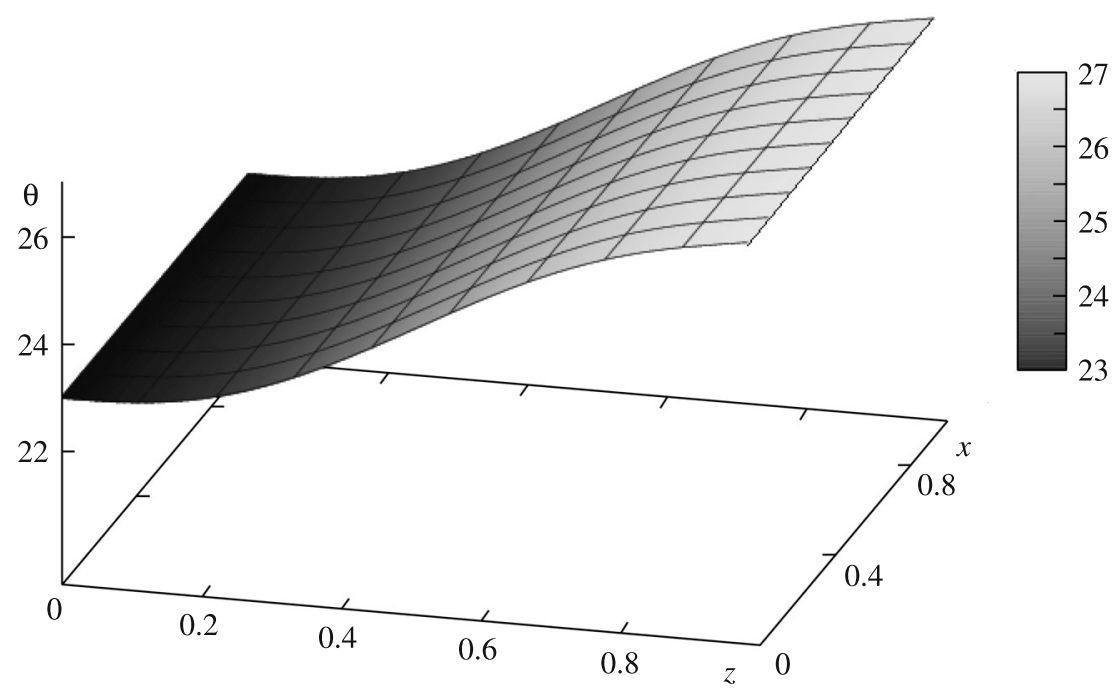

Fig. 3. Temperature distribution in $70 \%$ isopropanol aqueous solution.

the last equalities in (2.15) enables us to separate the problem of finding the functions $w(x)$ and $\alpha(x)$. For the constants we obtain a system of linear algebraic equations from which find the values $c_{1}, \ldots, c_{6}$, the constant $c_{7}$ remaining unknown by virtue of the restrictions on $\alpha(x)$ from (2.15).

In calculations we used the parameters for the mixture of water and aqueous solution of sodium chloride with concentration of 28.5 pro mil, which corresponds to the chemical composition of sea water. In Table 2 we present the physical parameters corresponding to the average temperature $12.5^{\circ} \mathrm{C}$, reestablished from the data of $[16,17]$ using the least squares method.

Figure 5 demonstrates the velocity distribution depending on the quantity $f_{0}$ from boundary condition (2.4), which corresponds to the heat flux from the lower wall. The higher $f_{0}$, the more intense the heat flux from the lower wall, which leads to a more intense mixing and, hence, to a change in flow direction. This can be seen from the velocity profiles shown in the figure. Curve 1 plotted for $f_{0}=0.0001$ demonstrates two flow zones, whereas curves 2 and 3 , corresponding to $f_{0}=0.001$ and 0.01 , respectively, display a more

Table 2

\begin{tabular}{c|c|c|c|c|c|c}
\hline$\rho_{0}, \mathrm{~kg} / \mathrm{m}^{3}$ & $v, \mathrm{~m}^{2} / \mathrm{s}$ & $\beta_{1}, 1 / \mathrm{K}$ & $\beta_{2}$ & $\chi, \mathrm{m}^{2} / \mathrm{s}$ & $D, \mathrm{~m}^{2} / \mathrm{s}$ & $D^{\theta}, \mathrm{m}^{2} /(\mathrm{Ks})$ \\
\hline 1020.32 & $1277 \times 10^{-9}$ & $1742 \times 10^{-7}$ & $-76 \times 10^{-5}$ & $106 \times 10^{-9}$ & $6106 \times 10^{-12}$ & $-172 \times 10^{-17}$ \\
\hline
\end{tabular}




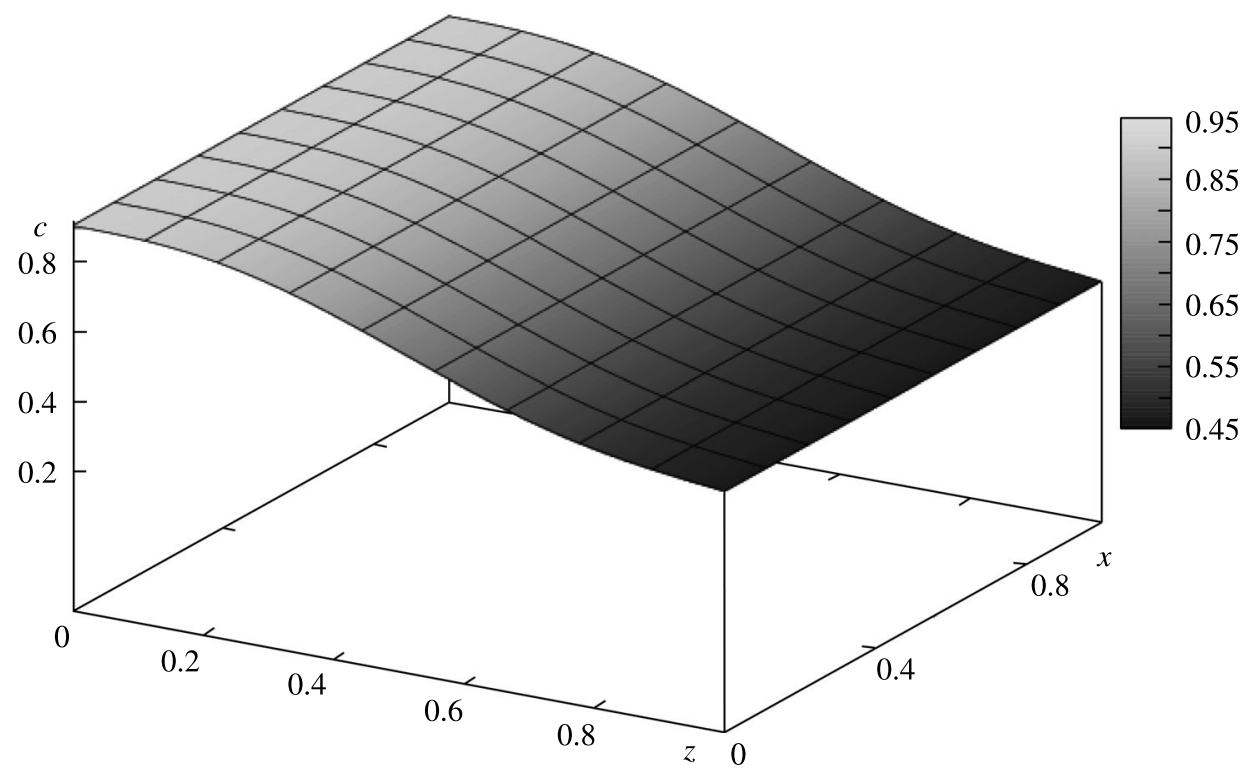

Fig. 4. Concentration distribution in isopropanol aqueous solution.

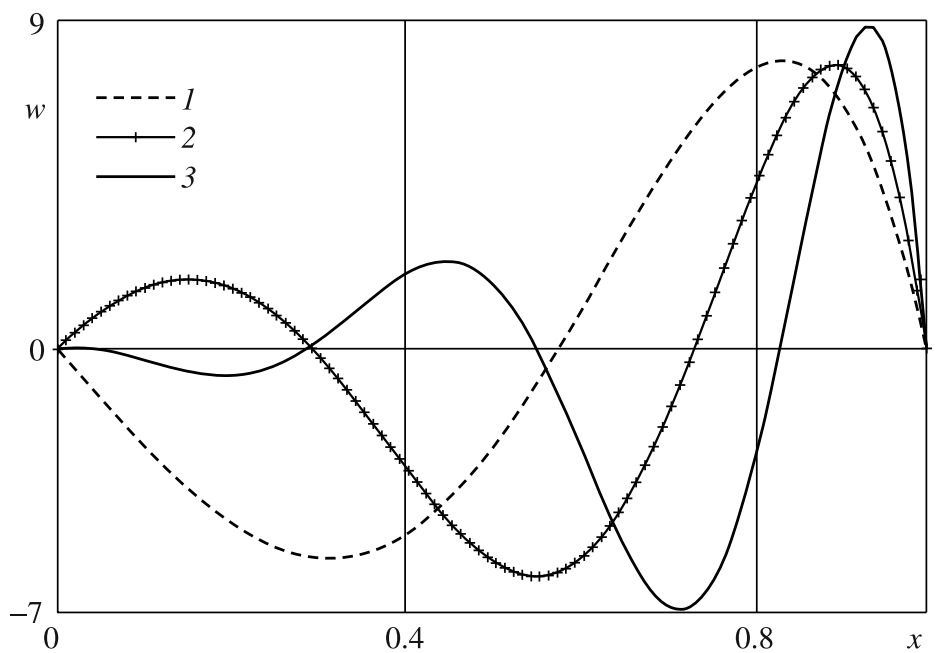

Fig. 5. Sodium chloride aqueous solution velocity profiles for different $f_{0}$ values.

frequent transition from direct to reverse flow zones and vice versa. Since the coefficient $D^{\theta}$ is here negative (see Table 2), normal thermodiffusion can be observed: the heavier sodium chloride moves into the less heated region and near the less heated wall $x=1$ the velocity is greater than anywhere in the layer.

Summary. The obtained solutions that describe unidirectional thermal diffusion flows are an important generalization of the well-known Birikh solution [2] to include the case of steady-state binary mixture flow. Restrictions on the temperature and concentration functions, for which the initial system of equations can be completely integrated, are written down. Formulations are proposed for boundary-value problems that describe the flow in the layer between rigid walls for the given law of temperature distribution over the walls or in the absence of heat flux across them. Restrictions on the physical characteristics of the mixture, for which the solution and/or boundary conditions have a simpler form, are found. Solution examples are constructed for two boundary-value problems that describe unidirectional flows of binary mixtures with linear and quadratic temperature distributions over the rigid walls. In view of nonlinearity and high order of the initial equations, the solutions obtained are of interest not only for possible physical applications but also as examples of exact solutions of equations of mixed type. For example, they can be used for checking the correctness of numerical methods. 
The work was supported financially by the Russian Foundation for Basic Research (project No. 14-0100067).

\section{REFERENCES}

1. G.A. Ostroumov, Free Convection under Inner Problem Conditions (Gostekhizdat, Moscow, 1952) [in Russian].

2. R.V. Birikh, "Thermocapillary Convection in a Horizontal Fluid Layer", Prikl. Mekh. Tekhn. Fiz., No. 3, 69-72 (1966).

3. M.K. Smith and S.H. Davis, "Instabilities of Dynamic Thermocapillary Liquid Layers. Pt. 1. Convective Instabilities," J. Fluid Mech.132, 119-144 (1983).

4. A.S. Ortiz-Pérez and L.A. Dávalos-Orozco, "Convection in a Horizontal Fluid Layer under an Inclined Temperature Gradient," Phys. Fluids 28 (3), 084107-084111 (2011).

5. V.K. Andreev, Birikh Solution of the Convection Equations and Its Certain Generalizations, Preprint 1-10 (Institute of Computational Modeling, Siberian Branch of the Russian Academy of Sciences, Krasnoyarsk, 2010) [in Russian].

6. V.B. Bekezhanova, "Change of the Types of Instability of a Steady Two-Layer Flow in an Inclined Channel," Fluid Dynamics 46 (4), 525-535 (2011).

7. V.V. Pukhnachev, "Nonstationary Analogs of the Birikh Solution," Proc. Altai State Univ. [in Russian], No. 1-2, 62-69 (2011).

8. S.N. Aristov and E.Yu. Prosviryakov, "Strayified Free Plane Convection Flows," Nelineynaya Dinamika 9 (4), 651-657 (2013).

9. V.K. Andreev and I.V. Stepanova, "Ostroumov-Birikh Solution of Convection Equations with Nonlinear Buoyancy Force," Appl. Math. Comput. 228, 59-67 (2014).

10. V.K. Andreev and I.I. Ryzhkov, "Group Classification and Exact Solutions of the Thermodiffusion Equations," Differents. Uravneniya 41 (4), 508-517 (2005).

11. I.I. Ryzhkov, "Invariant Solutions of the Binary Mixture Thermodiffusion Equations in the Case of Plane Flow," Prikl. Mekh. Tekhn. Fiz. 47 (1), 95-108 (2006).

12. I.I. Ryzhkov and I.V. Stepanova, "Group Properties and Exact Solutions for the Model of Vibrational Binary Mixture Convection,” Prikl. Mekh. Tekhn. Fiz. 52 (4), 72-82 (2011).

13. O.N. Goncharova and E.V. Rezanova, "Example of an Exact Solution of the Stationary Problem of Two-Layer Flow with Evaporation at the Interface, Prikl. Mekh. Tekhn. Fiz., No. 2, 68-79 (2014).

14. E. Kamke, Differentialgleichungen: Lösungsmethoden und Lösungen (Akademische Verlagsgesellschaft Geest \& Portig, Leipzig, 1956).

15. A. Mialdun, V. Yasnou, V. Shevtsova, et al., "A Comprehensive Study of Diffusion, Thermodiffusion, and Soret Coefficients of Water-Isopropanol Mixtures," J. Chem. Phys. 136 (244512), 1-13 (2012).

16. D.R. Caldwell, "Thermal and Fickian Diffusion of Sodium Chloride in a Solution of Oceanic Concentration," Deep-Sea Res. 20, 1029-1039 (1973).

17. M.H. Sharqawy, J.H. Lienhard, and S.M. Zubair, "Thermophysical Properties of Seawater: a Review of Existing Correlations and Data," Desalinat. Treatment 16, 354-380 (2010). 